\title{
Strain Sensitivity Enhancement in Suspended Core Fiber Tapers
}

\author{
Ricardo M. ANDRÉ ${ }^{1,2}$, Susana O. SILVA ${ }^{1,2}$, Martin BECKER ${ }^{3}$, Kay SCHUSTER ${ }^{3}$, \\ M. ROTHARDT ${ }^{3}$, H. BARTELT ${ }^{3}$, Manuel B. MARQUES ${ }^{1,2}$, and Orlando FRAZÃO ${ }^{1}$ \\ ${ }^{1}$ INESC Porto, Rua do Campo Alegre 687, 4169-007 Porto, Portugal \\ ${ }^{2}$ Departamento de Física e Astronomia da Faculdade de Ciências da Universidade do Porto, Rua do Campo Alegre 687, \\ 4169-007 Porto, Portugal \\ ${ }^{3}$ IPHT, Institute for Photonic Technology Jena, Albert-Einstein-Str. 9, 07745 Jena, Germany \\ *Corresponding author: Orlando FRAZÃOＥ-mail: ofrazao@inescporto.pt
}

\begin{abstract}
Suspended core fiber tapers with different cross sections (with diameters from $70 \mu \mathrm{m}$ to $120 \mu \mathrm{m})$ are produced by filament heating. Before obtaining the taper, the spectral behavior of the suspended core fiber is a multimode interference structure. When the taper is made, an intermodal interference between a few modes is observed. This effect is clearly visible for low taper core dimensions. Since the core and cladding do not collapse, two taper regions exist, one in the core and the other in the cladding. The cladding taper does not affect the light transmission, only the core is reduced to a microtaper. The spectral response of the microtaper based-suspended core fiber is similar to a beat of two interferometers. The strain is applied to the microtaper, and with the reduction in the transverse area, an increase in sensitivity is observed. When the taper is immersed in a liquid with a different index of refraction or subjected to temperature variations, no spectral change occurs.
\end{abstract}

Keywords: Optical fiber sensor, taper, microstructured fiber

\section{Introduction}

Optical fibers based on silica have become an irreplaceable asset in telecommunications, data transport and sensing. Low loss single mode fibers (SMFs) have revolutionized telecommunications but fall short when much smaller dimensions are needed for integrated photonic devices. Fiber tapering of conventional fibers to reduce the waveguide dimensions has been thoroughly investigated. The taper adiabaticity and shape have been extensively studied $[1,2]$. Many fiber tapering techniques have been developed such as pulling the fiber around a heated sapphire rod [3], $\mathrm{CO}_{2}$ lasers [4], and flame-brushing [5]. Low-loss tapers with subwavelength diameters offer empowering optical and mechanical properties such as strong confinement and consequent non-linear interactions [6], large evanescent fields [5], and high mechanical strength [7].

In the last 15 years, microstructured optical fibers have attracted a lot of attention due to their excellent properties. Over the last decade, the tapering of microstructured optical fibers has been perfected [8, 9], and very interesting optical microstructures have been developed. It was shown that microstructured optical fibers could be tapered while retaining their cross-sectional profiles with

Received: 2 February 2012 / Revised version: 18 May 2012

(C) The Author(s) 2012. This article is published with open access at Springerlink.com 
little or no distortion [10]. The cross-sectional profile could thus be scaled down proportionally producing structures with very small core diameters. Magi et al. [10] were the first to achieve a pitch below $300 \mathrm{~nm}$ in a tapered holey fiber. Tapered holey fiber sensors as temperature-independent strain sensors were also reported [11]. Tapered microstructured optical fibers have also been employed for the efficient generation of the supercontinuum [6].

In this paper, the tapering of a suspended core fiber (SCF) is investigated. In particular, an SCF with a small core diameter was tapered down reducing even more its diameter. Tapers of different cross-sections were produced by filament heating and were characterized in the strain, temperature, and external index of refraction.

\section{Experimental results and discussion}

Figure 1 illustrates the experimental setup used to produce and characterize the suspended core fiber tapers. The tapers were produced using a VYTRAN-glass processing workstation. The VYTRAN (GPX-3000 series) is a glass processing platform that performs fusion splicing and tapering of specialty fibers. The system consists of a filament heater, precision stages with multi-axis control (fiber holding blocks) and a microscopic high resolution CCD imaging system. The filament heater has a wide temperature range (from $100{ }^{\circ} \mathrm{C}$ to $3000{ }^{\circ} \mathrm{C}$ ) which allows the fusion and processing of various fiber types and sizes. The working principle is based on heating a portion of the fiber in the filament heater to its softening point while applying a tensile force by pulling the fiber with the holding blocks. As the fiber is elongated, its cross sectional area will be reduced accordingly. Taper properties can be ascertained by means of a graphic interface. The tapers were fabricated in the microstructured fiber (IPHT) with $8.4-\mu \mathrm{m}$ and $128-\mu \mathrm{m}$ core and cladding diameters, respectively. This fiber had four holes with diameters of $22 \mu \mathrm{m}$ each and a bridge width of
$1.5 \mu \mathrm{m}$. 10-mm-length tapers were made in SCF sections that in turn were spliced between two single-mode fibers (SMF28). A broadband source in the 1550-nm spectral range and an optical spectrum analyzer were used to monitor in real time the tapering process. A similar experimental setup was used to characterize the SCF tapers with increasing strain. But in this case a setup without the filament heater was used.

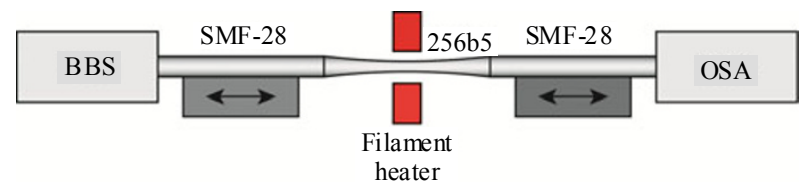

Fig. 1 Experimental setup.

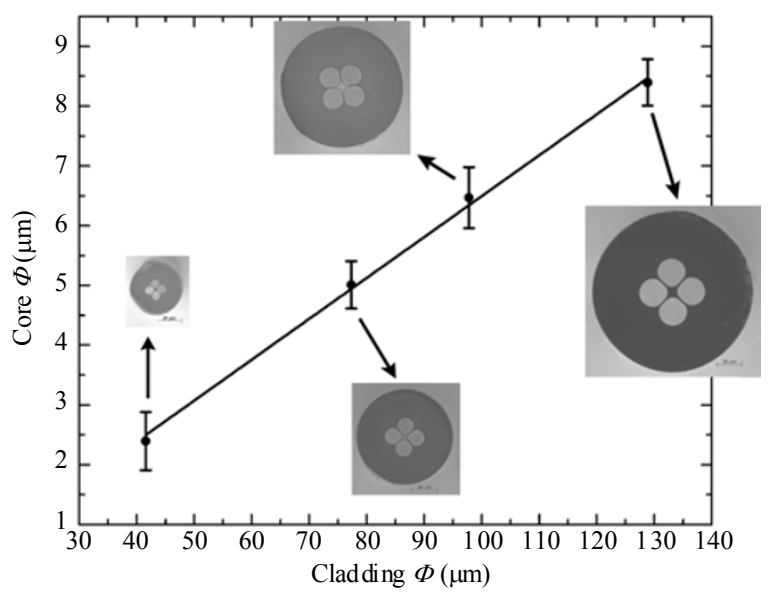

Fig. 2 Relationship between core and cladding diameters.

One can observe from Fig. 2 that when the original SCF is tapered, both core and cladding diameters are reduced. It should be noted that the core and cladding have proportional reductions but the cross sectional profile is maintained. Even at $32 \%$ of the original size, the holes do not collapse. The core geometry is maintained all through the process, and therefore, the waveguide suffers a uniform reduction. The ratio between diameters of the core and cladding is $0.069 \pm 0.002$. The tapers are also immersed in a liquid with a refractive index of 1.36 , and no change in the spectral response occurs, confirming that the holes did not collapsed. Figure 2 also shows a photo of the taper region which confirms this behavior. Before tapering, the sensing 
head acts as a multimode interference devicethe light field propagating along the input SMF enters the SCF section, and several modes are excited. This interference appears because different modes, which propagate along the SCF with different phase velocities, are recombined at the splice between the single mode fiber and SCF, and in this case, a well-defined notch peak at about 1585 $\mathrm{nm}$ appears (as shown in Fig.3(a)). After tapering, intermodal interference appears, as shown in Fig. 3(b). This occurs due to the reduction in the core diameter and consequent reduction in the number of propagating modes. Figure 3(b) shows a new spectral response when compared with Fig.3(a). A beat signal appears due to the combination of two or

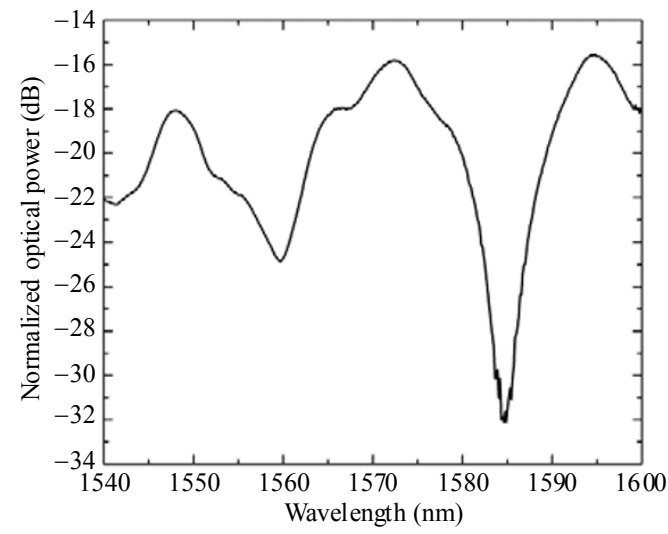

(a)

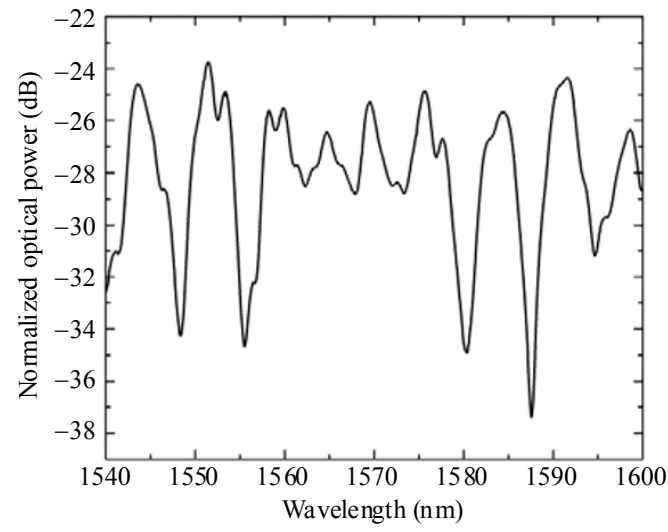

(b)

Fig. 3 Spectral responses (a) before and (b) after fabricating the taper in the suspended core fiber.

more interferometers. These interferometers are combined in the splice but are created in two different regions namely in the tapered and untapered regions.

For strain characterization, the sensor heads were placed on micrometric translation stages and subjected to controlled stretching. Of the total length of the fiber on which strain was applied (approximately $135 \mathrm{~mm}$ ), only the section from $25 \mathrm{~mm}$ to $30 \mathrm{~mm}$ was made up of suspended core fiber. Of the SCF section, only about $10 \mathrm{~mm}$ were tapered to different diameters.

In Fig. 4, different strain sensitivities for the $80-\mu \mathrm{m}$ and $120-\mu \mathrm{m}$ SCF tapers are clearly identifiable. Figure 5 shows that the strain sensitivity increases when the taper diameter decreases. This behavior is expected [12]. The sensing head is composed by an SCF section combined with a fused taper. The strain gauge of the sensing head has a total length of $L_{\text {total }}=L_{\text {taper }}+L_{\text {fiber. }}$. If strain $\left(\varepsilon_{\text {taper }}\right)$ is applied to the taper structure, at constant temperature, the wavelength will be shifted by $\Delta \lambda_{\text {taper }}$ according to

$$
\Delta \lambda_{\text {taper }}=\kappa_{0} \varepsilon_{\text {taper }}
$$

where $\kappa_{0}$ is a constant characteristic of the fiber material, which can be easily determined experimentally by analyzing the variation of the wavelength as a function of the strain of the untapered SCF at constant temperature.

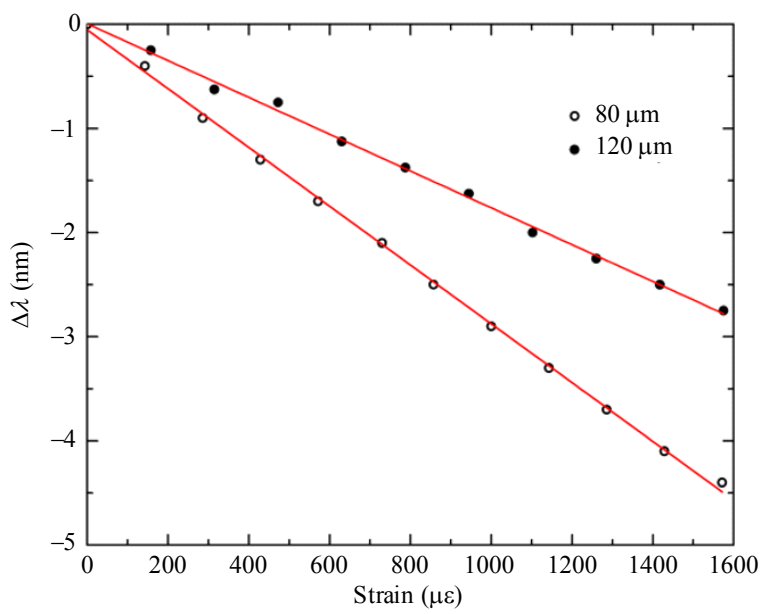

Fig. 4 Wavelength change with the applied strain for $80-\mu \mathrm{m}$ and $120-\mu \mathrm{m}$ SCF tapers. 
However, if the strain is applied to the entire sensor, then an unequal load of the stress will appear along each section of the sensor depending on the mechanical resistance. In particular, the strain loads applied to the SCF and the fused taper are related according to

$$
\varepsilon_{\text {fiber }} E A_{\text {fiber }}=\varepsilon_{\text {taper }} E A_{\text {taper }}
$$

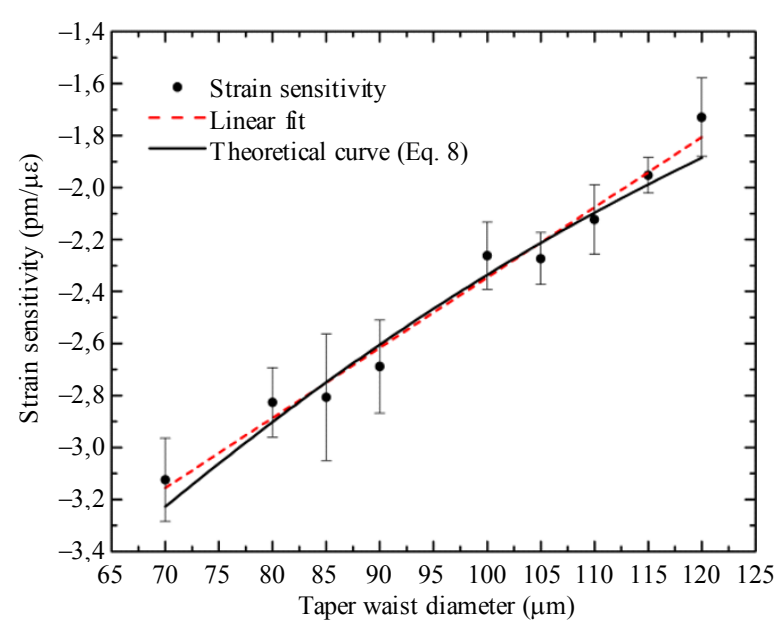

Fig. 5 Relationship between the strain sensitivity and the taper waist.

where $E$ is the Young modulus of the sensor material, and $A_{i}$ ( $i=$ fiber or taper) is the cross-sectional area of the suspended core fiber in the untapered and tapered regions, respectively. It is assumed that the mechanical properties of the material in both suspended core fiber and taper regions are the same. Consequently, the strain applied to the two sensor sections and the cladding diameters are related according to

$$
\frac{\varepsilon_{\text {fiber }}}{\varepsilon_{\text {taper }}}=\frac{d_{\text {taper }}^{2}}{d_{\text {fiber }}^{2}}
$$

where $d_{\text {fiber }}$ and $d_{\text {taper }}$ are the cladding diameters of the SCF and taper, respectively. The longitudinal strain of the fused taper and the piece of the fiber are given by

$$
\varepsilon_{\text {taper }}=\frac{\Delta L_{\text {taper }}}{L_{\text {taper }}} \quad \text { and } \quad \varepsilon_{\text {fiber }}=\frac{\Delta L_{\text {fiber }}}{L_{\text {fiber }}}
$$

where $L_{\text {taper }}$ and $L_{\text {fiber }}$ are the lengths of the sensor sections, and $\Delta L_{\text {taper }}$ and $\Delta L_{\text {fiber }}$ are the extensions of the taper and the fiber, respectively. By definition, the total longitudinal strain of the sensing head is given by

$$
\varepsilon=\frac{\Delta L_{\text {taper }}+\Delta L_{\text {fiber }}}{L_{\text {taper }}+L_{\text {fiber }}} .
$$

This approximation is valid when considering a sensing head composed of fibers with different diameters. Nevertheless, in the case of tapered fibers, this approximation can be very accurate, even more so if the taper transitions are short when compared with the taper length. In this case, even though the VYTRAN splicing machine provides long transition regions, it also provides a long taper with a uniform diameter.

By combining (3)-(5), it is possible to derive the relationship between the strains applied to the whole sensor as

$$
\varepsilon_{\text {taper }}=\frac{L_{\text {fiber }}+L_{\text {taper }}}{L_{\text {fiber }} \frac{d_{\text {taper }}^{2}}{d_{\text {fiber }}^{2}}+L_{\text {taper }}} \varepsilon_{0}
$$

where $\varepsilon_{0}$ is the total strain applied to the structure. By substituting (6) into (1), the wavelength shift can be rewritten as

$$
\Delta \lambda_{\text {taper }}=\kappa_{0} \frac{L_{\text {fiber }}+L_{\text {taper }}}{L_{\text {fiber }} \frac{d_{\text {taper }}^{2}}{d_{\text {fiber }}^{2}}+L_{\text {taper }}} \varepsilon_{0}
$$

from which one can obtain the strain sensitivity of the whole sensing head

$$
\kappa=\kappa_{0} \frac{L_{\text {fiber }}+L_{\text {taper }}}{L_{\text {fiber }} \frac{d_{\text {taper }}^{2}}{d_{\text {fiber }}^{2}}+L_{\text {taper }}} .
$$

The final strain sensitivity is in reality given by the strain sensitivity of the untapered fiber $\left(\kappa_{0}\right)$ affected by a factor that is greater than one for the tapered fiber $\left(d_{\text {taper }}<d_{\text {fiber }}\right)$. As $d_{\text {taper }}$ becomes smaller, this multiplying factor becomes larger, and therefore the strain sensitivity increases. All this is confirmed by the results shown in Fig. 5. The obtained mean 
strain sensitivity to the taper size determined was $(2.7 \pm 0.1) \times 10^{-2}$ strain $^{-1}$. From $120 \mu \mathrm{m}$ $(-1.73 \mathrm{pm} / \mu \varepsilon)$ to $80 \mu \mathrm{m}(-2.83 \mathrm{pm} / \mu \varepsilon)$, the strain sensitivity increased 64\% (as shown in Fig. 4). Also that plotted in Fig. 5 is the theoretical estimate given by (8). From this, it is possible to estimate $\kappa_{0}$ to be $-1.7 \mathrm{pm} / \mu \varepsilon$. This approximation is within experimental error which confirms the applicability of the previous strain sensitivity deduction to these tapers.

The SCF tapers were also subjected to temperature variations, but no sensitivity to such variations was observed. Figure 6 presents the spectral response of the $70-\mu \mathrm{m}$ SCF taper at $30{ }^{\circ} \mathrm{C}$ and $70{ }^{\circ} \mathrm{C}$ where no discernable wavelength shift can be detected. This result is expected due to the properties of the SCF. The fiber is made of pure silica, and its thermo-optic coefficient is very low.

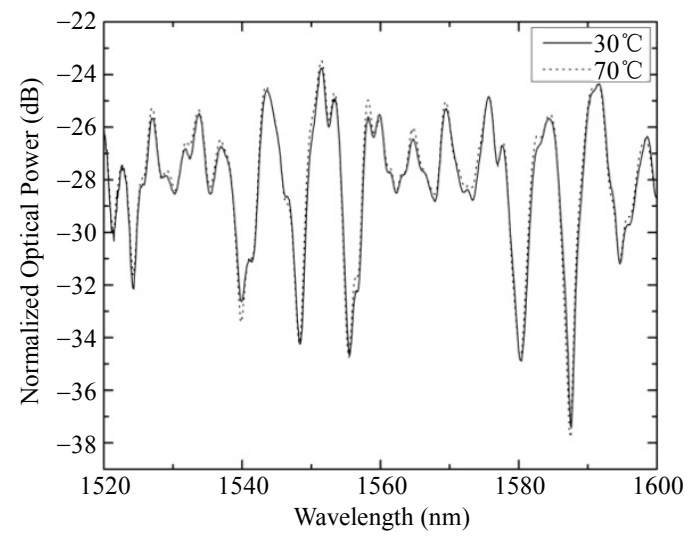

Fig. 6 Temperature dependence of the $70-\mu \mathrm{m}$ SCF taper.

\section{Conclusions}

To summarize, tapers from $70 \mu \mathrm{m}$ to $120 \mu \mathrm{m}$ were produced on an originally $125-\mu \mathrm{m}$ SCF and characterized. They were deemed to have very low temperature and external refractive index sensitivities but extremely high sensitivity to the applied strain. Sensitivities of $-1.73 \mathrm{pm} / \mu \varepsilon$ and $-3.12 \mathrm{pm} / \mu \varepsilon$ for $120-\mu \mathrm{m}$ and $70-\mu \mathrm{m}$ taper structures, respectively, were obtained. An increase in the strain sensitivity with a decreasing taper diameter was observed. It was also verified that even when the SCF was tapered down to $40 \mu \mathrm{m}$, the holes did not collapse.

These tapered suspended core fiber structures have advantages when compared to normal fiber tapers as sensing elements. Besides having enhanced the strain sensitivity, they also have very low temperature sensitivity and are protected from external medium perturbations due to the strong confinement of light in an air-silica waveguide.

\section{Acknowledgement}

The authors would like to thank Fundação Calouste Gulbenkian for the grant provided under the award "Prémio de Estímulo à Investigação" attributed to Ricardo Melo André.

Open Access This article is distributed under the terms of the Creative Commons Attribution License which permits any use, distribution, and reproduction in any medium, provided the original author(s) and source are credited.

\section{References}

[1] J. D. Love, W. M. Henry, W. J. Stewart, R. J. Black, S. Lacroix, and F. Gonthier, "Tapered single-mode fibers and devices .I. adiabaticity criteria," IEE Proceeding J, vol. 138, no. 5, pp. 343-354, 1991.

[2] T. A. Birks and Y. W. Li, "The shape of fiber tapers," Journal of Lightwave Technology, vol. 10, no. 4, pp. 432-438, 1992.

[3] L. M. Tong, R. R. Gattass, J. B. Ashcom, S. L. He, J. Y. Lou, M. Y. Shen, I. Maxwell, and E. Mazur, "Subwavelength-diameter silica wires for low-loss optical wave guiding," Nature, vol. 426, no. 6968, pp. 816-8192003.

[4] T. E. Dimmick, G. Kakarantzas, T. A. Birks, and P. S. Russell, "Carbon dioxide laser fabrication of fused-fiber couplers and tapers," Applied Optics, vol. 38, no. 33, pp. 6845-6848, 1999.

[5] G. Brambilla, V. Finazzi, and D. J. Richardson, 
"Ultra-low-loss optical fiber nanotapers," Optics Express, vol. 12, no. 10, pp. 2258-2263, 2004.

[6] S. G. Leon-Saval, T. A. Birks, W. J. Wadsworth, P. S. J. Russell, and M. W. Mason, "Supercontinuum generation in submicron fiber waveguides," Optics Express, vol. 12, no. 13, pp. 2864-2869, 2004.

[7] F. Xu, P. Horak, and G. Brambilla, "Optical microfiber coil resonator refractometric sensor," Optics Express, vol. 15, no. 12, pp. 7888-7893, 2007.

[8] J. K. Chandalia, B. J. Eggleton, R. S. Windeler, S. G. Kosinski, X. Liu, and C. Xu, "Adiabatic coupling in tapered air-silica microstructured optical fiber," IEEE Photonics Technology Letters, vol. 13, no. 1, pp. 52-54, 2001.

[9] S. T. Huntington, J. Katsifolis, B. C. Gibson, J. Canning, K. Lyytikainen, J. Zagari, L. W. Cahill, and J. D. Love, "Retaining and characterising nano-structure within tapered air-silica structured optical fibers," Optics Express, vol. 11, pp. 98-104, 2003.

[10] E. C. Magi, P. Steinvurzel, and B. J. Eggleton, "Tapered photonic crystal fibers," Optics Express, vol. 12, no. 5, pp. 776-784, 2004.

[11] J. Villatoro, V. P. Minkovich, and D. Monzón-Hernández, "Temperature-independent strain sensor made from tapered holey optical fiber," Optics Letters, vol. 31, no. 3, pp. 305-307, 2006.

[12] O. Frazao, S. F. O. Silva, A. Guerreiro, J. L. Santos, L. A. Ferreira, and F. M. Araújo, "Strain sensitivity control of fiber Bragg grating structures with fused tapers," Applied Optics, vol. 46, no. 36, pp. 8578-8582, 2007. 